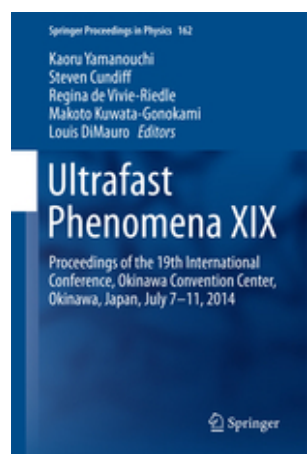

(C) 2015 Springer International Publishing Switzerland This article was published in: Ultrafast Phenomena XIX

Edited by: Kaoru Yamanouchi, Steven Cundiff, Regina de Vivie-Riedle, Makoto Kuwata-Gonokami, and Louis DiMauro.

Springer Proceedings in Physics 162, 813 - 816. (2015)

ISBN: 978-3-319-13241-9

DOI: $10.1007 / 978-3-319-13242-6 \_199$

Available online at:

http://link. springer.com/chapter/10.1007/978-3-319-13242-6_199 


\title{
The Influence of Generalized Focusing on Polarization-shaped Few-cycle Pulsed Beams
}

\author{
Balázs Major, Miguel A. Porras, Attila P. Kovács and Zoltán L. Horváth
}

\begin{abstract}
In this work we show how generalized focusing affects the polarizationstate of polarization-shaped pulsed beams being only a few cycles long. We present simulations to exemplify how this phenomenon can cause the rotation of the socalled 'polarization gating' pulse commonly used for isolated attosecond pulse generation. The general validity of the recently published simple rules for these diffraction-caused changes is also demonstrated.
\end{abstract}

\section{Introduction}

It is well-known that the polarization state of the statistically-stationary radiation of partially coherent or partially polarized electromagnetic sources can change on freespace propagation [1,2]. On the other hand, for deterministic, fully coherent and fully polarized pulsed beams used in experiments nowadays [3, 4, 5], such changes are only expected for beams having inhomogeneous polarization [6], or being tightly focused $[6,7]$. However, it has been recently shown that focusing affects the polarization state of such pulsed beams, if they are only few cycles long $[8,9]$. Here we summarize some properties of this phenomenon, and present an example to show how these findings can affect the polarization state of few-cycle pulses used in the various fields of science.

Balázs Major $(\bowtie) \cdot$ Attila P. Kovács · Zoltán L. Horváth

Department of Optics and Quantum Electronics, University of Szeged

Dóm tér 9, Szeged H-6720, Hungary

e-mail: bmajor@titan.physx.u-szeged.hu

Miguel A. Porras

Departamento de Física Aplicada a los Recursos Naturales and Grupo de Sistemas Complejos, Universidad Politécnica de Madrid

Rios Rosas 21, Madrid ES-28003, Spain 


\section{Focusing-induced polarization-state changes}

Similarly to Refs. [8, 9], we describe the polarization state of a polarization-shaped few-cycle pulse by its instantaneous polarization ellipse. In this description, at every moment of time a polarization ellipse is associated with the electric field in the same way as in the case of monochromatic waves, and the polarization state is primarily defined by the orientation $\psi(t)$ and ellipticity $\chi(t)$ of the instantaneous ellipse. Following the calculation given in Ref. [9], based on a general model of ideal focusing with only two perpendicular components of the electric field propagating independently, it can be shown that the instantaneous properties of the polarization state change according to the following approximate rules while the pulse having $\omega_{0}$ carrier angular frequency propagates from the focusing element to the focal point (in this model the same as propagating to the far field):

$$
\begin{gathered}
\psi^{(f)}(\tau)-\psi(\tau) \simeq \frac{1}{\omega_{0}} \frac{1}{1-\tan ^{2} \chi(\tau)} \frac{\mathrm{d} \tan \chi(\tau)}{\mathrm{d} \tau}, \\
\tan \chi^{(f)}(\tau)-\tan \chi(\tau) \simeq \frac{1}{\omega_{0}}\left[1-\tan ^{2} \chi(\tau)\right] \frac{\mathrm{d} \psi(\tau)}{\mathrm{d} \tau},
\end{gathered}
$$

where $\tau$ is the local time, which takes into account the time needed for the pulse to propagate from the source to the point of interest [9]. The expressions above show that the time-variation of the instantaneous ellipticity (orientation) induces a change in the instantaneous orientation (ellipticity) during focusing. This effect is demonstrated to be caused by diffraction, that is by the finite size of the source [9], or in other words by the Wolf effect [10]. As the temporal variation of $\psi(t)$ or $\chi(t)$ can only originate from the fast change of the phase difference or the rapid variation of the envelope ratio of the orthogonal components [8], this effect is more pronounced for few-cycle pulses, and disappear for monochromatic waves.

\section{An example: the polarization gating pulse}

In Ref. [9] the polarization gating (PG) pulse was an example to demonstrate (1) independently from (2). In this paper we expand this simple model of the PG pulse by taking into account the pulse chirp. Fig. 1(a) depicts the temporal evolution of the electric field in the case of the chirped PG pulse. For better visualization of the polarization state, the Stokes parameters of the instantaneous polarization ellipse are plotted in Fig. 1(b) for the initial and focused pulses. From this figure it is easy to notice that the primary effect of focusing is a constant rotation of the instantaneous ellipse, due to its time varying ellipticity (see (1)). However, it is important to note that due to the temporal chirp, the initial orientation is also time-dependent (linearly), but the effect in this case for this parameter is almost negligible. Also note that in case of zero chirp, the orientation would be time-independent, and in Fig. 1(b) the curves would go along the lines of longitude of the Poincare sphere. In 


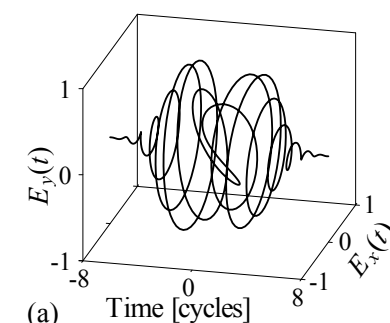

(a)

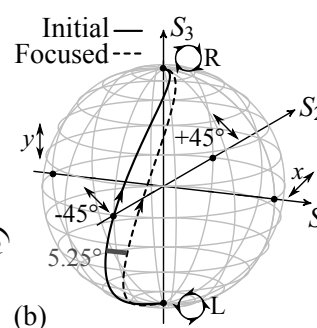

(b)

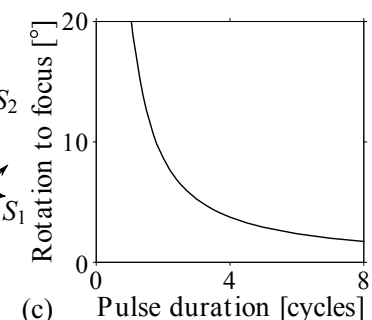

Fig. 1 (a) The $800 \mathrm{~nm}$ chirped polarization gating pulse acquired by sending a three-cycle pulse (in intensity-FWHM $\Delta T$ ) through two quarter-wave plates (as described in [4]). The quadratic term of temporal phase is: $\exp \left(i 0.3 t^{2} / \Delta T^{2}\right)$. Based on the simple model of the PG pulse in Ref. [9]. (b) Representation of the instantaneous polarization ellipse of the polarization gating pulse on the Poincare sphere. The continuous curve represents the initial, the dashed curve the focused pulse. The arrows indicate the time evolution. (c) Scaling of the rotation effect with pulse duration.

Fig. 1(c) it is shown how this effect, which is independent of focal length or beam size, disappears for longer and is enhanced for close to single-cycle pulses.

\section{Conclusions}

We have shown that focusing in general affects the polarization state of polarizationshaped few-cycle electromagnetic pulsed beams, and these changes are caused by diffraction. We demonstrated our findings with the chirped polarization gating pulse.

Acknowledgements The project was partially funded by TÁMOP-4.2.2.A-11/1/KONV-20120060 "Impulse lasers for use in materials science and biophotonics", supported by the European Union and co-financed by the European Social Fund. M. A. P. acknowledges financial support from Project MTM2012-39101-C02-01 of Ministerio de Economa y Competitividad of Spain.

\section{References}

1. D.F.V. James, J. Opt. Soc. Am. A 11(5), 1641 (1994)

2. O. Korotkova, E. Wolf, Opt. Commun. 246(1-3), 35 (2005)

3. F. Weise, S. Weber, M. Plewicki, A. Lindinger, Chem. Phys. 332(2-3), 313 (2007)

4. I.J. Sola, A. Zaïr, R. López-Martens, P. Johnsson, K. Varjú, E. Cormier, J. Mauritsson, A. L'Huillier, V. Strelkov, E. Mével, E. Constant, Phys. Rev. A: At., Mol., Opt. Phys. 74(1), $013810(2006)$

5. C. Ruchert, C. Vicario, C.P. Hauri, Phys. Rev. Lett. 110(12), 123902 (2013)

6. L.E. Helseth, Phys. Rev. E: Stat., Nonlinear, Soft Matter Phys. 72(4), 047602 (2005)

7. C. Spindler, W. Pfeiffer, T. Brixner, Appl. Phys. B: Lasers Opt. 89(4), 553 (2007)

8. M.A. Porras, J. Opt. Soc. Am. B 30(6), 1652 (2013)

9. B. Major, M.A. Porras, Z.L. Horváth, J. Opt. Soc. Am. A 31(6), 1200 (2014)

10. Z. Dačić, E. Wolf, J. Opt. Soc. Am. A 5(7), 1118 (1988) 